\title{
Clinicopathological Significance of Maspin Expression in Breast Cancer
}

\begin{abstract}
Maspin is a unique serine proteinase inhibitor that has tumor suppressor activity. It has been reported that maspin is expressed in normal human mammary epithelial cells and it is down-regulated during the progression of cancer. However, to date, there is very limited data on the clinical significance of maspin expression in human breast cancer. In this study, maspin expression was assessed immunohistochemically from 80 invasive ductal carcinoma (IDC) specimens of the breast. Also, maspin expression was compared with the clinicopathological factors (age, grade, tumor size and lymph node status), the expression of estrogen receptor (ER), progesterone receptor (PR) and p53, DNA ploidy and the overall survival in an attempt to assess its prognostic value. The maspin expression was positive in 25 IDC cases (31.3\%). The maspin expression in IDC was significantly correlated with a higher histologic grade, a larger tumor size, a positive p53 status and shorter survival. There was an inverse association with maspin expression and the PR status. These findings suggest that maspin expression is not down-regulated with the progression of cancer and maspin expression may be associated with a poor prognosis. The immunohistochemical detection of maspin in breast cancers may be helpful for predicting an aggressive phenotype.
\end{abstract}

Key Words : Serpins; Immunohistochemistry; Prognosis; Breast Neoplasms
Mi-Ja Lee, Chae-Hong Suh, Zhu-hu Li ${ }^{*}$

Department of Pathology, College of Medicine, Chosun University, Gwangju, Korea; Department of Pathology ${ }^{*}$, College of Medicine, Yanbian University, Jilin, China

Received : 18 March 2005

Accepted : 16 September 2005

Address for correspondence

Mi-Ja Lee, M.D.

Department of Pathology, College of Medicine, Chosun University, 375 Suhsukdong, Dong-gu, Gwangju 501-759, Korea

Tel : +82.62-230-6345, Fax : +82.62-234-4584

E-mail : mjblee@chosun.ac.kr

${ }^{*}$ This study was supported by research funds from Chosun University, 2001.

\section{INTRODUCTION}

The factors that regulate the cell-matrix interactions are essential for invasiveness and metastatic behavior. Proteinases that are expressed by epithelial cells, such as cathepsins, metalloproteinases and serine proteinases, are able to degrade the extracellular matrix and so they may facilitate tumor invasion (1).

Maspin is a relatively novel member of the serine proteinase inhibitor (serpin) family; it plays a role in the development of the mammary gland (2) and it shows positive expression on myoepithelial cells and normal secretory epithelial cells (3). In vitro and animal models have shown that maspin inhibits tumorigenesis, which consists of tumor cell growth, invasion and metastasis (3, 4). Zhang et al. (5) reported that maspin also inhibits endothelial cell motility and angiogenesis, and they provided possible evidence of a maspin-tumor suppressor mechanism.

Although the precise molecular and biologic mechanisms of maspin remain unknown, there is an evidence that maspin interacts with the $\mathrm{p} 53$ tumor suppressor pathway and it may function as an inhibitor of angiogenesis in vitro and in vivo $(5,6)$. Zou et al. (6) reported that $\mathrm{p} 53$ regulates the expression of maspin, and that its expression can be induced by DNA damage in cells containing the wild-type p53. However, to date, the exact tumor suppressor mechanism of maspin, as related with $\mathrm{p} 53$, remains unknown.
There is very limited data on the clinical significances of maspin expression in human breast cancer. There has been one report investigating the prognostic significance of the maspin expression in a large series involving breast cancer patients with long-term follow-up (7). The prognostic utility of maspin currently remains controversial (8-10).

The aim of this study was to investigate the role of maspin in tumorigenesis and tumor progression, and we wanted to determine its potential as a new prognostic factor for breast carcinomas. We tried to assess the maspin expression in invasive ductal carcinoma (IDC). By comparing with the clinicopathologic variables (age, histologic grade, tumor size, and lymph node status), the estrogen receptor (ER), progesterone receptor $(\mathrm{PR})$ and $\mathrm{p} 53$ expression, and DNA ploidy, we attempted to determine the clinicopathological significance of maspin expressions in breast carcinomas. Furthermore, we examined the prognostic value of maspin expression by establishing its correlation with the survival rates of the patients.

\section{MATERIALS AND METHODS}

\section{Materials}

Eighty patients with IDC of the breast who were operated on at our hospital between 1991 and 1996 were considered 
for analysis. We excluded those breast tissues from patients who had no follow up and if they had undergone chemotherapy or radiation therapy prior to surgery. The patients had undergone either a mastectomy or breast-conserving surgery with axillary lymph node (LN) dissection. The patients who underwent mastectomy had received adjuvant chemotherapy and hormonal therapy. The patients who underwent breastconserving surgery had received adjuvant radiation therapy and hormonal therapy. By reviewing the medical records and the pathological reports of the selected patients, we summarized the patient's age, tumor size and lymph node status. We determined the survival rates for the 80 IDC patients. The patients were followed up for 11-115 months (median 63 months) postoperatively.

\section{Pathological classification}

The histologic grades of the invasive ductal carcinomas were determined according to the Nottingham modification of the Bloom-Richardson system (11). According to the TNM classification, the primary tumor sizes were divided into tumors smaller than $2.0 \mathrm{~cm}$ (T1), tumors between 2.1 to $5.0 \mathrm{~cm}$ (T2) and tumors larger than $5.0 \mathrm{~cm}$ (T3). Lymph node involvement was divided into no metastasis and metastasis.

\section{Immunohistochemistry}

Tissue sections were prepared as $4 \mu \mathrm{m}$-thick-slides from the paraffin-embedded specimens; they were deparaffinized with xylene, rehydrated with alcohol and washed with distilled water. For antigen retrieval, the slides were immersed in citrate buffer solution (10 mM, pH 6.0) and microwaved for $10 \mathrm{~min}$ at $99^{\circ} \mathrm{C}(900 \mathrm{~W})$, and then they were cooled and washed in Tris buffer solution (TBS). The endogenous peroxidase activity was blocked with $3 \%$ hydrogen peroxide. They were reacted with primary antibodies and allowed to incubate overnight at $4{ }^{\circ} \mathrm{C}$. The primary antibodies were monoclonal antibodies for maspin (EAW24, 1:20, NeoMarkers, Fremont, CA, U.S.A.), ER (ER-1D5, 1:50, Immunotech, Westbrook, ME, U.S.A.), PR (clone 1A6, 1:50, Immunotech) and p53 (DO-7, 1:50, NeoMarkers). This was followed by using a Picture $^{\mathrm{TM}}$ Plus kit (a polymer detection system, Zymed, South San Francisco, CA, U.S.A.). AEC (3-amino-9-ethyl carbazole) solution was applied as the chromogen and the nuclei were counterstained with hematoxylin. For the positive control of each antibody, prostate tissues were used for maspin and breast carcinoma tissues that had been previously identified as being positive were used for ER, PR, and p53.

\section{Evaluation}

The positivity for each antibody was determined as a definite positive reaction in the cytoplasm or cytoplasm and nucleus for maspin, and a definite positive reaction in the nucle- us for each ER, PR, and p53 protein. The results were determined as negative for each antibody if less than $10 \%$ staining was noted, and the results were positive if more than $10 \%$ staining was noted.

\section{Analysis of DNA ploidy}

For the DNA index analysis, two or three serial paraffin sections were prepared at $50 \mu \mathrm{m}$-thickness; they were deparaffinized with xylene, rehydrated with alcohol and washed with PBS (phosphate buffered saline solution). The tissue sections were minced to a single cell suspension in DMSO buffer (dimethylsulfoxide in sucrose sodium citrate buffer). $2 \times 10^{6}$ cells were washed twice with PBS. The cells were reacted with solution A (trypsin in spermine tetrahydrochloride detergent buffer, CycleTEST ${ }^{\text {тм }}$ Plus Reagent Kit, Becton-Dickinson, Franklin Lakes, NJ, U.S.A.) for $10 \mathrm{~min}$ at room temperature. After treatment with solution B (RNase A and trypsin inhibitor in spermine buffer) for $10 \mathrm{~min}$ at room temperature, the cells were stained with solution $\mathrm{C}$ (propidium iodide in spermine buffer). The DNA index analysis was determined by a FACSCalibur (Becton-Dickinson) and with using human lymphocyte nuclei as the controls.

\section{Statistical analysis}

Pearson chi square tests were used to evaluate the relationship among the patients age, histological grade, tumor size, lymph node status, DNA ploidy and expressions of maspin, $\mathrm{ER}, \mathrm{PR}$ and $\mathrm{p} 53$. Univariate survival analyses were carried out using log-rank tests. Multivariate survival analyses were carried out according to the Cox regression model. Significance was determined as $p$ value less than 0.05 . All statisti$\mathrm{cal}$ analyses were performed using the SPSS program (version 12.0).

\section{RESULTS}

\section{Expressions of maspin, ER, PR and p53}

The maspin expression showed strong nuclear reactivity and weak cytoplasmic reactivity on the myoepithelial cells in the normal gland neighboring the ductal carcinoma in situ (DCIS) (Fig. 1) and in the IDC (Fig. 2). No positive staining was observed in the epithelial cells. However, in the cancer cells, the maspin was expressed in the cytoplasm. Some cells also exhibited maspin nuclear staining that accompanied the cytoplasmic staining (Fig. 3). The maspin expression was positive in 25 IDC cases (31.3\%). The expressions of ER, PR and p53 in the IDC tissues were positive in 42 cases (52.5\%), 37 cases $(46.3 \%)$, and 26 cases $(32.5 \%)$, respectively. 


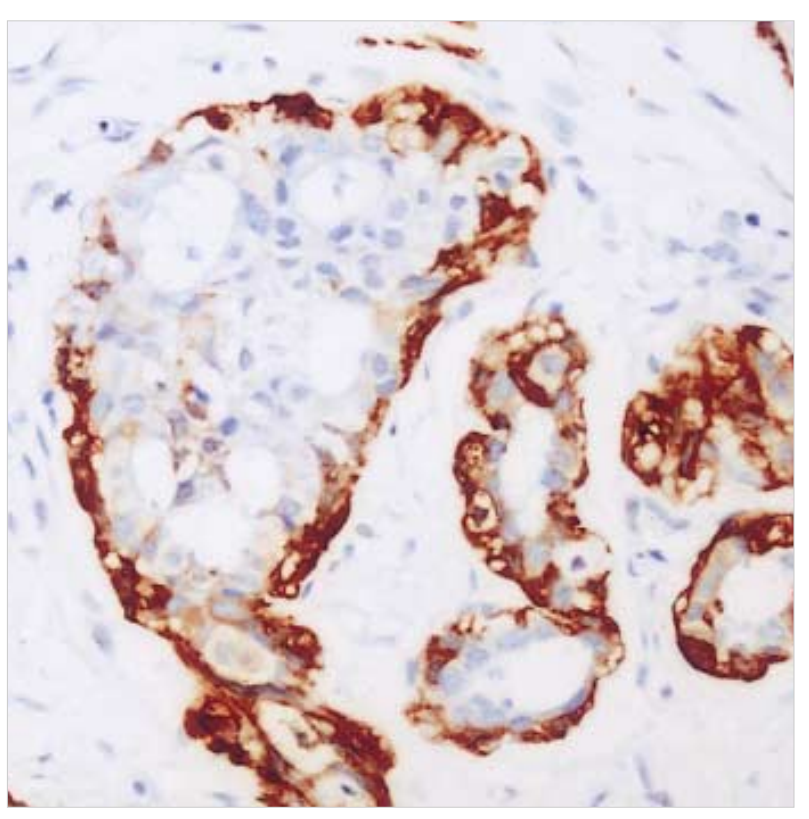

Fig. 1. The maspin expression shows strong nuclear reactivity and weak cytoplasmic reactivity on the myoepithelial cells of ductal carcinoma in situ and remaining glands. The epithelial cells are negatively stained (Anti-maspin, ×200).

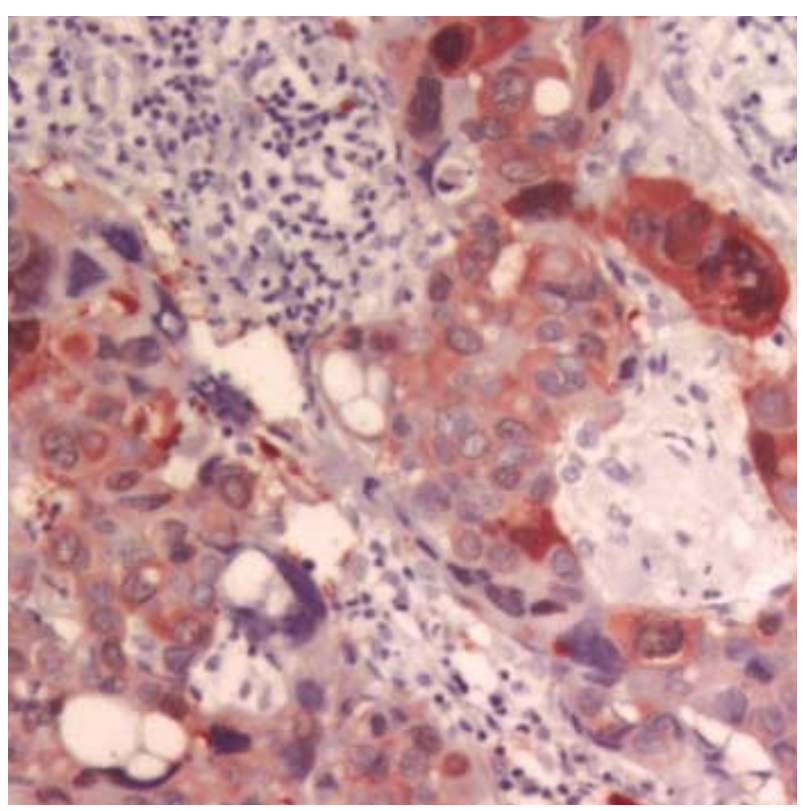

Fig. 3. The maspin is expressed in the cytoplasm of the cancer cells. Some cells shows nuclear staining that accompanied the cytoplasmic staining (Anti-maspin, $\times 400$ ).

\section{Correlation between maspin positivity and the clinicopathological variables}

When the maspin expression was compared with the clinicopathological variables, the maspin expression showed a significant association with the higher histologic grade $(p=$

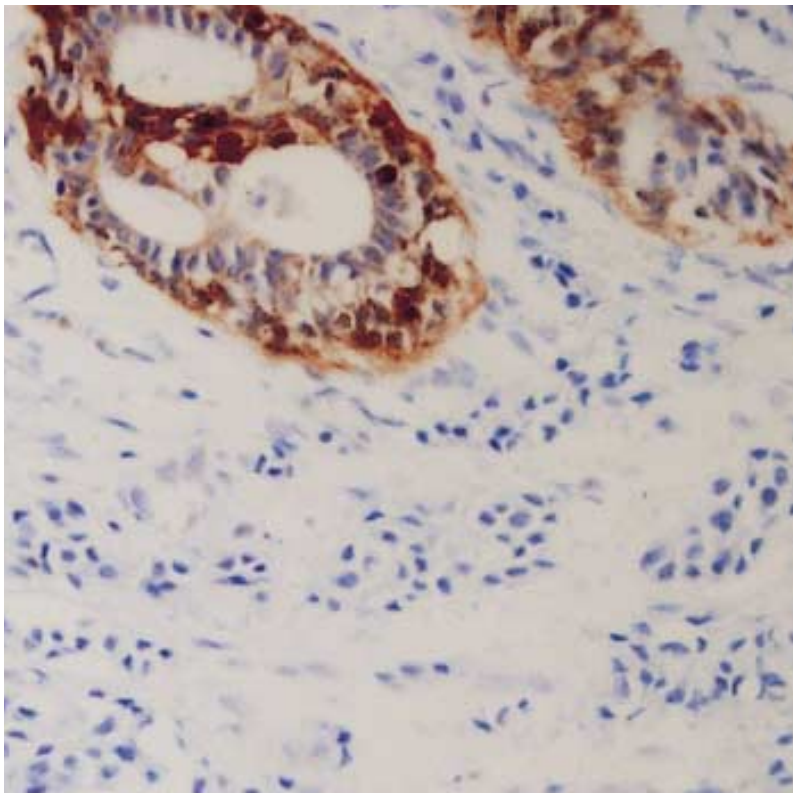

Fig. 2. Immunonegativity for maspin is seen in invasive ductal carcinoma, but strong positivity in myoepithelial cells of the remaining glands (Anti-maspin, ×200).

Table 1. Correlation between maspin positivity and clinicopathological variables in invasive ductal carcinoma of breast

\begin{tabular}{|c|c|c|c|c|}
\hline & \multirow{2}{*}{ Total } & \multicolumn{2}{|c|}{ Maspin expression } & \multirow{2}{*}{$p$ value } \\
\hline & & Positive & Negative & \\
\hline Cases & 80 & 25 & 55 & \\
\hline Age (yr) & & & & 0.95 \\
\hline$\leq 39$ & 15 & 5 & 10 & \\
\hline $40-49$ & 29 & 10 & 19 & \\
\hline $50-59$ & 25 & 7 & 18 & \\
\hline$\geq 60$ & 11 & 3 & 8 & \\
\hline Grade & & & & 0.005 \\
\hline I & 18 & 1 & 17 & \\
\hline$\|$ & 37 & 11 & 26 & \\
\hline III & 25 & 13 & 12 & \\
\hline Tumor size $(\mathrm{cm})$ & & & & 0.02 \\
\hline$\leq 2.0$ & 19 & 2 & 17 & \\
\hline $2.1-5.0$ & 51 & 17 & 34 & \\
\hline$>5.0$ & 10 & 6 & 4 & \\
\hline Lymph nodes & & & & 0.695 \\
\hline Negative & 41 & 12 & 29 & \\
\hline Positive & 39 & 13 & 26 & \\
\hline
\end{tabular}

$0.005)$ and the larger tumor size $(p=0.02)$ (Table 1).

\section{Correlation between maspin expression and expression of ER, PR and p53, and DNA ploidy}

There was a significant inverse relationship between the maspin and PR expressions $(p=0.001)$. In other words, while the $\mathrm{PR}$ was positive, the maspin was negative and when the PR was negative, the maspin was positive. Also, a significant 
Table 2. Correlation between maspin expression and expression of ER, PR and p53, and DNA ploidy in invasive breast cancer

\begin{tabular}{|c|c|c|c|c|c|}
\hline & \multirow{2}{*}{ Total } & \multicolumn{3}{|c|}{ Maspin expression } & \multirow{2}{*}{$p$ value } \\
\hline & & & & Negative & \\
\hline \multirow[t]{2}{*}{ ER } & Positive & 42 & 11 & 31 & 0.30 \\
\hline & Negative & 38 & 14 & 24 & \\
\hline \multirow[t]{2}{*}{ PR } & Positive & 37 & 5 & 32 & 0.001 \\
\hline & Negative & 43 & 20 & 23 & \\
\hline \multirow[t]{2}{*}{ p53 } & Positive & 26 & 12 & 14 & 0.046 \\
\hline & Negative & 54 & 13 & 41 & \\
\hline \multirow[t]{3}{*}{ DNA } & Ploidy & & & & 0.398 \\
\hline & Dipoidy & 68 & 20 & 48 & \\
\hline & Aneuploidy & 12 & 5 & 7 & \\
\hline
\end{tabular}

Table 3. Cox multivariate analysis of overall survival in invasive breast cancer

\begin{tabular}{llcc}
\hline Variables & $p$ value & $\mathrm{RR}$ & $95 \% \mathrm{Cl}$ \\
\hline Age & 0.56 & 1.12 & $0.77-1.62$ \\
Histologic grade & 0.006 & 2.12 & $0.24-3.64$ \\
Tumor size & 0.01 & 2.13 & $1.14-3.98$ \\
Positive nodes & 0.0003 & 4.50 & $1.99-10.15$ \\
ER & 0.03 & 0.44 & $0.21-0.92$ \\
PR & 0.0001 & 0.14 & $0.06-0.37$ \\
Maspin & 0.01 & 2.52 & $1.25-5.12$ \\
p53 & 0.005 & 2.72 & $1.34-5.52$ \\
Aneuploidy & 0.25 & 1.69 & $0.69-4.13$ \\
\hline
\end{tabular}

$\mathrm{RR}$, relative risk; $95 \% \mathrm{Cl}, 95 \%$ confidence interval.

association was noted between the maspin and p53 expressions in the IDC tissue ( $p=0.046)$ (Table 2).

\section{Correlation between the survival rate and the variables}

Eighty IDC patients were followed up for at least five years, and their survival rate was $61.3 \%$. Univariate analysis showed a significant difference in the overall survival according to the histologic grade $(p=0.01)$, tumor size $(p=0.04)$, the lymph node status ( $p=0.0001)$, the ER status $(p=0.02)$, the PR status $(p<0.00001)$, the p53 status $(p=0.003)$ and the maspin expression ( $p=0.007$ ) (Fig. 4). Age and DNA ploidy showed no significant difference for the overall survival. From the Cox multivariate analyses, the histologic grade $(p=0.006)$, tumor size $(p=0.01)$, positive nodes $(p=0.0003)$, the expressions of ER ( $p=0.03), \operatorname{PR}(p=0.0001)$, maspin $(p=0.01)$, and p53 ( $p=$ 0.005 ) were prognostically significant (Table 3 ).

\section{DISCUSSION}

Proteinases and their inhibitors are known to play important roles in tumor invasion and metastasis. Two classes of proteinases have been extensively studied in breast cancer and other cancers; serine proteinase and their inhibitors (plasminogen activator inhibitors, PAI) and the metalloproteinases and

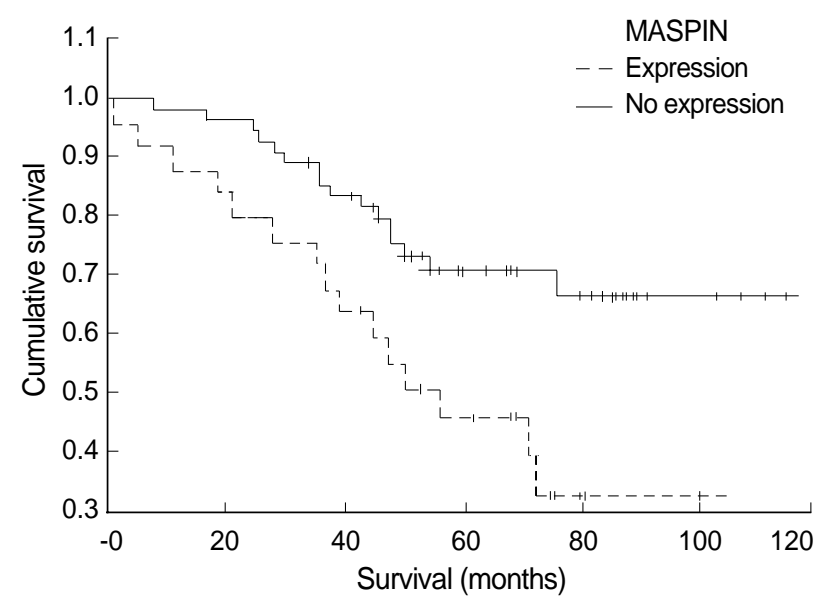

Fig. 4. Univariate analysis of 80 patients of invasive ductal carcinoma shows a significant difference in the overall survival according to the maspin expression.

the tissue inhibitors of metalloproteinases $(12,13)$. As a member of the serpin superfamily, maspin (mammary serpin) is a novel proteinase inhibitor that is related to other inhibitors such as PAI (13).

Since the first report by Zou et al. (3) revealed the loss of maspin expression in advanced cancers with using immunohistochemistry (IHC), there had been a few reports investigating the clinical significance of maspin expression by performing IHC. Hojo et al. (14) have found that maspin-positive breast cancer showed a low pathologic grade of malignancy. However, in their study, the sample number was small $(n=49)$ and any correlation with the clinical outcome was not investigated. Maass et al. (15) have reported that the downregulation of the maspin gene in breast carcinoma was associated with a higher risk of distant metastasis by using RTPCR. However, since the mammary myoepithelial cells surrounding the normal ducts and the noninvasive ductal components express the maspin protein (16), it could be difficult to evaluate the "maspin expression in breast carcinoma" by means of employing RT-PCR methods. Maass et al. (17) have recently suggested that the decline in the expression of maspin was associated with tumor progression in ductal carcinomas of the breast with using 12 cases of DCIS, 128 cases of IDC and their lymph node metastases. However, in their study, there was a weak inverse association with the PR status and the number of DCIS cases was small $(n=12)$.

Their findings strongly contrast with ours. We demonstrated that the expression of maspin, as detected by IHC, was significantly correlated with an aggressive phenotype that was characterized by a higher histologic grade, a larger tumor size and a negative PR status in the breast carcinoma tissues. The univariate analysis via the log-rank test clearly indicated a strong correlation between the expression of maspin and a shorter overall survival. Moreover, the multivariate analysis that included such known prognostic factors as tumor size, histologic grade, hormone receptor and p53 status showed 
that the expression of maspin was an independent indicator of a poor prognosis. These findings seemed to be contrary to the expected results that the loss of maspin expression would correlate with a poor prognosis if the expression of maspin was down-regulated during the progression of breast cancer.

A recent studies showed that the expression of maspin was correlated with an aggressive phenotype in the breast cancer $(7,8)$, pancreatic cancer (18) and ovarian cancer (19). Also, the maspin expression was significantly associated with a shorter relapse-free survival and a shorter overall survival (7). Umekita et al. (7) results were similar with ours. The association between the maspin expression level and the survival rate has not been demonstrated in human cancer except in the reports by Umekita et al. (7) and Xia et al. (20). Umekita et al. (8) suggested three possible explanations for the aberrant expression of maspin in breast cancer cells. One was the mutation of the maspin gene, the second was a high intracellular concentration of maspin resulting in auto-inhibition of its activity. The third was that myoepithelial cell differentiation in carcinoma cells could contribute to the more aggressive phenotype. We think that the mutation of the maspin gene causes the loss of normal function of the maspin protein. Therefore, mutational analyses with using maspin-positive samples are needed to resolve this issue.

Maspin expression has always regarded to be limited to the cytoplasmic compartment of the cell. However the presence of maspin in both the nuclear and cytoplasmic compartments has been shown by Pemberton et al. (21) with using cell fractions and Western blotting, and by Chu et al. (22) with using IHC. One possible explanation for the detection of signals from both nucleus and cytoplasm with using IHC is the antigen retrieval technique; this might have exhibited a crossreacting epitope in the nucleus. Mohsin et al. (9) reported that maspin nuclear staining was significantly associated with good prognostic factors, while cytoplasmic staining was associated with poor prognostic markers. However, the function and significance of this pattern of expression is currently unknown. In our study, the staining in normal breast tissue (this was present in some areas of the tumor) showed a strong nuclear and weak cytoplasmic staining in the myoepithelial cells, but there was cytoplasmic staining or cytoplasmic and nuclear staining in the tumor cells. We decided to evaluate the maspin expression in the cytoplasm or the cytoplasm and nuclei of breast cancer cells.

The mechanism underlying the function of maspin as a tumor suppressor is not fully understood. Hojo et al. (14) reported that the maspin positive group of tumors had a tendency to stain less strongly for $\mathrm{p} 53$ protein in the tumor sections. They considered that maspin can be upregulated by promotor activation through p53 directly binding to the $\mathrm{p} 53$ consensus binding site in the maspin promotor, but the $\mathrm{p} 53$ binding site was refractory to mutant $\mathrm{p} 53$. Zhang et al. (23) indicated that in most tumors mutant $\mathrm{p} 53$ is inversely correlated with maspin expression, and this suggests maspin is likely a p53 target gene in vivo. Our study and Umekita et al. (7) revealed a significant correlation between the expression of maspin and the $\mathrm{p} 53$ protein in breast cancer by performing IHC. Although our data cannot definitely prove this functional correlation between both proteins, it may provide additional information on a p53 dependent regulatory pathway of the maspin protein in breast cancer.

Although a larger series and longer follow-up are needed, our present investigation suggests that the expression of maspin could be an independent poor prognostic indicator for breast cancer patients. Further investigation are needed to clarify the precise mechanism of the aberrant expression of maspin in the breast cancer that has an aggressive phenotype.

\section{REFERENCES}

1. Duffy MJ, McCarthy K. Matrix metalloproteinases in cancer: prognostic markers and targets for therapy (review). Int J Oncol 1998; 12: 1343-8.

2. Zhang M, Magit D, Botteri F, Shi HY, He K, Li M, Furth P, Sager R. Maspin plays an important role in mammary gland development. Dev Biol 1999; 215: 278-87.

3. Zou Z, Anisowicz A, Hendrix MJ, Thor A, Neveu M, Sheng S, Rafidi K, Seftor E, Sager R. Maspin, a serpin with tumor-suppressing activity in human mammary epithelial cells. Science 1994; 263: 526-9.

4. Sheng S, Carey J, Seftor EA, Dias L, Hendrix MJ, Sager R. Maspin acts at the cell membrane to inhibit invasion and motility of mammary and prostatic cancer cells. Proc Natl Acad Sci USA 1996; 93: 11669-74.

5. Zhang M, Volpert O, Shi YH, Bouck N. Maspin is an angiogenesis inhibitor. Nat Med 2000; 6: 196-9.

6. Zou Z, Gao C, Nagaich AK, Connell T, Saito S, Moul JW, Seth P, Appella E, Srivastava S. p53 regulates the expression of the tumor suppressor gene maspin. J Biol Chem 2000; 275: 6051-4.

7. Umekita Y, Ohi Y, Sagara Y, Yoshida H. Expression of maspin predicts poor prognosis in breast-cancer patients. Int J Cancer 2002; 100: 452-5.

8. Umekita Y, Yoshida H. Expression of maspin is up-regulated during the progression of mammary ductal carcinoma. Histopathology 2003; 42: 541-5.

9. Mohsin SK, Zhang M, Clark GM, Craig Allred D. Maspin expression in invasive breast cancer: association with other prognostic factors. J Pathol 2003; 199: 432-5.

10. Ito Y, Yoshida H, Tomoda C, Uruno T, Takamura Y, Miya A, Kobayashi K, Matsuzuka F, Matsuura N, Kuma K, Miyauchi A. Maspin expression is directly associated with biological aggressiveness of thyroid carcinoma. Thyroid 2004; 14: 13-8.

11. Elston CW, Ellis IO. Pathological prognostic factors in breast cancer. I. The value of histological grade in breast cancer: experience from a large study with long-term follow-up. Histopathology 1991; 19; 403-10.

12. Duffy MJ, Reilly D, O'Sullivan C, O'Higgins N, Fennelly JJ, Andreasen P. Urokinase-plasminogen activator, a new and independent 
prognostic marker in breast cancer. Cancer Res 1990; 50: 6827-9.

13. Potempa J, Korzus E, Travis J. The serpin superfamily of proteinase inhibitors: structure, function, and regulation. J Biol Chem 1994; 269: 15957-60.

14. Hojo T, Akiyama Y, Nagasaki K, Maruyama K, Kikuchi K, Ikeda $\mathrm{T}$, Kitajima M, Yamaguchi K. Association of maspin expression with the malignancy grade and tumor vascularization in breast cancer tissues. Cancer Lett 2001; 171: 103-10.

15. Maass N, Hojo T, Rosel F, Ikeda T, Jonat W, Nagasaki K. Down regulation of the tumor suppressor gene maspin in breast carcinoma is associated with a higher risk of distant metastasis. Clin Biochem 2001; 34: 303-7.

16. Sternlicht MD, Kedeshian P, Shao ZM, Safarians S, Barsky SH. The human myoepithelial cell is a natural tumor suppressor. Clin Cancer Res 1997; 3: 1949-58.

17. Maass N, Teffner M, Rosel F, Pawaresch R, Jonat W, Nagasaki K, Rudolph P. Decline in the expression of the serine proteinase inhibitor maspin is associated with tumour progression in ductal carcinomas of the breast. J Pathol 2001; 195: 321-6.

18. Maass N, Hojo T, Ueding M, Luttges J, Kloppel G, Jonat W, Nagasaki K. Expression of the tumor suppressor gene Maspin in human pan- creatic cancers. Clin Cancer Res 2001; 7: 812-7.

19. Sood AK, Fletcher MS, Gruman LM, Coffin JE, Jabbari S, KhalkhaliEllis Z, Arbour N, Seftor EA, Hendrix MJ. The paradoxical expression of maspin in ovarian carcinoma. Clin Cancer Res 2002; 8: 292432.

20. Xia W, Lau YK, Hu MC, Li L, Johnston DA, Sheng S, El-Naggar A, Hung MC. High tumoral maspin expression is associated with improved survival of patients with oral squamous cell carcinoma. Oncogene 2000; 19: 2398-403.

21. Pemberton PA, Tipton AR, Pavloff N, Smith J, Erickson JR, Mouchabeck ZM, Kiefer MC. Maspin is an intracellular serpin that partitions into secretory vesicles and is present at the cell surface. J Histochem Cytochem 1997; 45: 1697-706.

22. Chu YC, Park IS, Kim YJ, Kim JM, Han HS, Han JY, Kim YB. Expression of maspin protein in ductal hyperplasia, intraductal carcinoma and invasive ductal carcinoma of the breast. Korean J Pathol 1999; 33: 614-9.

23. Zhang W, Zhang M. Tissue microarray analysis of maspin expression and its reverse correlation with mutant $p 53$ in various tumors. Int J Oncol 2002; 20: 1145-50. 